\title{
Near-field evolution in strongly pumped broad area diode lasers
}

\author{
Martin Hempel*a, Jens W. Tomm ${ }^{\mathrm{a}}$, Martina Baeumler ${ }^{\mathrm{b}}$, Helmer Konstanzer ${ }^{\mathrm{b}}$, Jayanta Mukherjee ${ }^{\mathrm{c}}$, \\ Thomas Elsaesser ${ }^{\mathrm{a}}$ \\ ${ }^{a}$ Max-Born-Institut, Max-Born-Strasse 2A, 12489 Berlin, Germany; \\ ${ }^{\mathrm{b}}$ Fraunhofer Institut für Angewandte Festkörperphysik, Tullastrasse 72, 79108 Freiburg, \\ Germany \\ ${ }^{\mathrm{c}}$ Advanced Technology Institute, University of Surrey, Guildford, GU2 7XH, UK
}

\begin{abstract}
Many applications such as pumping of solid state lasers or ignition of explosives require high optical output powers during a short period. Pulsed operated diode lasers meet these requirements. They can be driven at elevated power levels, well above the ones specified for continuous wave (cw) operation. The optical near-field intensity of a diode laser in this operation regime is a key parameter since it determines the beam properties of the device. High power AlGaAs/GaAs quantum well broad area diode lasers are subjected to single pulse step tests carried out up to and beyond their ultimate limits of operation. Laser near-fields are monitored on a picosecond time scale using a streak-camera setup during pulse currents of up to $\sim 50$ times the threshold current. A transition from gain guiding to thermally-induced index guiding of the near-field is shown. Further power increase is prevented by catastrophic optical damage (COD). This sudden failure mechanism is studied in conjunction with filamentary properties of the near-field. The defect growth dynamics resolved on the picosecond time scale is used to gather inside into the physics behind COD.
\end{abstract}

Keywords: high power broad area diode lasers, near-field, filamentation, degradation, catastrophic optical damage

\section{INTRODUCTION}

A variety of applications like pumping solid state lasers ${ }^{1}$, igniting explosives ${ }^{2}$, and replacing conventional spark plugs in cars call for high optical power densities during a short period of time. These requirements are fulfilled by semiconductor diode lasers driven in short pulse regime well above the pumping level specified for continuous wave (cw) operation. Laser operation at such high powers is beneficial, e.g., to reduce the number of pump lasers necessary in a system in order to reduce size and costs. ${ }^{3}$

A key parameter of diode lasers is the optical near-field intensity (NF) since it determines the beam properties and is therefore relevant for the optics coupled to the laser source. Its behavior, especially the temporal evolution, under extremely high pumping current is still not well understood. Up to now NF dynamics are investigated up to 23 times lasing threshold for ridge waveguide lasers ${ }^{4}$ and only up to $\sim 2$ times lasing threshold for broad area (BA) lasers. ${ }^{5}$ With the ongoing development of the semiconductor laser technology much higher pumping levels can be achieved. In the present work we investigate the NF of BA lasers at driving currents $I$ of up to $\sim 50$ times lasing threshold.

Operation at even higher power levels is hampered by the catastrophic optical damage (COD). COD is a sudden failure mechanism affecting diode lasers emitting at high optical powers. This process starts at a certain pumping level, the socalled COD threshold. This term can be used to indicate the output power level (or the current) at which COD occurs. Since the lasers used in this study are all from one batch, the relation between optical output power and current is fixed and the term will be used in the following for the current. At this threshold a critical temperature $T_{\text {crit }}$ of $120^{\circ} \mathrm{C}-$ $160^{\circ} \mathrm{C}$,see Ref. ${ }^{6}$, is reached in a small volume at the laser facet making this region highly absorbing for the laser light. This leads to a fast temperature rise by transferring energy from the light field to the damage site, called thermal runaway. In this report we investigate the connection between the NF filamentation properties, COD, and the transient defect dynamics with picoseconds time resolution.

*hempel@mbi-berlin.de; phone +49(0)30/6392 1453; fax +49(0)30/6392 1459; www.mbi-berlin.de 


\section{EXPERIMENTAL}

A batch of commercial $808 \mathrm{~nm}$ edge emitting high-power BA diode lasers is analyzed. The gain-guided AlGaAs/GaAs quantum well $(\mathrm{QW})$ structure is mounted $p$-side down with the epitaxial side close to the heat sink. The emitter stripe has a width and length of $50 \mu \mathrm{m} \times 1.4 \mathrm{~mm}$, respectively. The specified $\mathrm{cw}$ current is $(0.65 \pm 0.01)$ A while emitting $(0.57 \pm 0.02) \mathrm{W}$, with a threshold current of $(0.21 \pm 0.01) \mathrm{A}$. The front and rear facets have standard low reflective and high reflective coatings, respectively. The used devices are labeled A, B, C . . for clarity. The experimental data shown in this article are restricted to four representative devices A-D.

In the experimental setup the laser is attached directly to the pulsed laser driver PicoLAS LDP-V 50-100 V3 to guarantee a short current rise time of $\sim 10 \mathrm{~ns}$. The laser temperature is stabilized to $(23.0 \pm 0.2)^{\circ} \mathrm{C}$ using a thermo-electric cooler. The near-field is imaged via a $25 \times$ microscope objective to the input slit of a HAMAMATSU streak camera C1587 (equipped with single sweep unit M1953), resulting in a spatial mapping of $\sim 240 \mathrm{~nm}$ per camera pixel.

With this setup we use the so-called step tests with a fixed pulse length of $300 \mathrm{~ns}$. This strategy follows the scheme presented in Fig. 1. Starting from a current of $I_{0}=1.6 \mathrm{~A}$, significantly below the COD threshold for this pulse length, we apply a single current pulse to the device. During this the NF and the current passing through the device is monitored. To assure that the device is not even partly damaged a test pulse with a low current (due to technical reasons it is equivalent to $I_{0}$ here) is applied and the NF recorded during that is compared to the one which is recorded under the same conditions before the device test starts. If a clear sign for a COD event is visible the test cycle can either be stopped for further analysis in this early damage stage or the COD can be re-ignited be applying further pulses with the same current. If no COD occurs the current is increased by a step as small as $0.5 \mathrm{~A}$ and a new test cycle is entered. For a more detailed description see Refs. ${ }^{7,8}$

COD is manifested by a rapid change of the NF accompanied by a growing region of substantially lowered intensity, also seen in a sudden output power drop. Furthermore the NF in the test pulse changes with respect to the undamaged device case. Additionally one can see a clearly damaged front facet with an optical microscope.

The advantage of this approach is that the preparation of very early damage phases (with an output power loss of $10 \%$ or less at a single emitter) becomes possible at different pulse length, as shown in Ref. ${ }^{9}$ The COD process can be timesliced by stopping the degradation at the pulse end and reviving it during the subsequent pulse, which we call $C O D$ reignition. This concept was verified earlier. ${ }^{8}$

During our experiments we stopped the above described testing cycle after a varying number of COD re-ignitions. A part of the tested batch was opened afterwards by mechanically removing the $n$-contact and selective etching of the GaAs substrate and buffer layer. ${ }^{10}$ After this preparation the QW plane is mapped by micro-photoluminescence $(\mu \mathrm{PL})$.

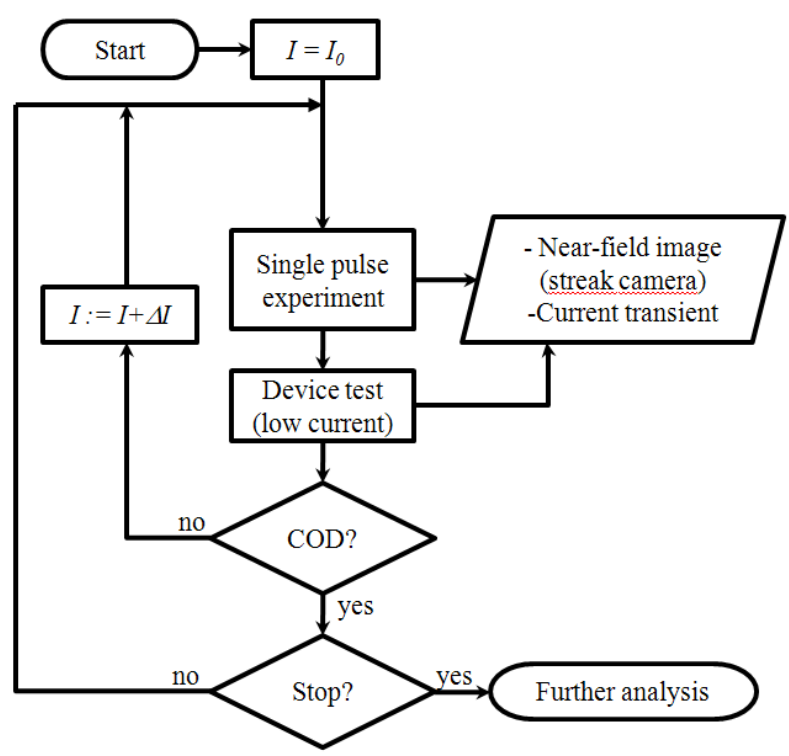

Figure 1. Scheme of the used device testing cycle denoted as step test. 


\section{RESULTS}

\subsection{Near-field at high operation currents}

Figure 2 shows the evolution of the NF of device A in a single $300 \mathrm{~ns}$ pulse for $I=6.0 \mathrm{~A}(\sim 29 \times$ threshold current), $7.5 \mathrm{~A}$ $(36 \times)$, and $8.5 \mathrm{~A}(41 \times)$. The temporal resolution in the left column is $\sim 675 \mathrm{ps}$ while in the other subfigures a value of $\sim 18$ ps is reached. Their temporal position is indicated in the corresponding overview image at the left side. The change of the NF pattern with increasing current and time is obvious. Cuts through the NF at different times, each averaged over $\sim 8$ ns, are shown in Fig. 3. The inset shows the area under the NF cut curves, which stays almost constant over time. This area is proportional to the optical output power of the laser.

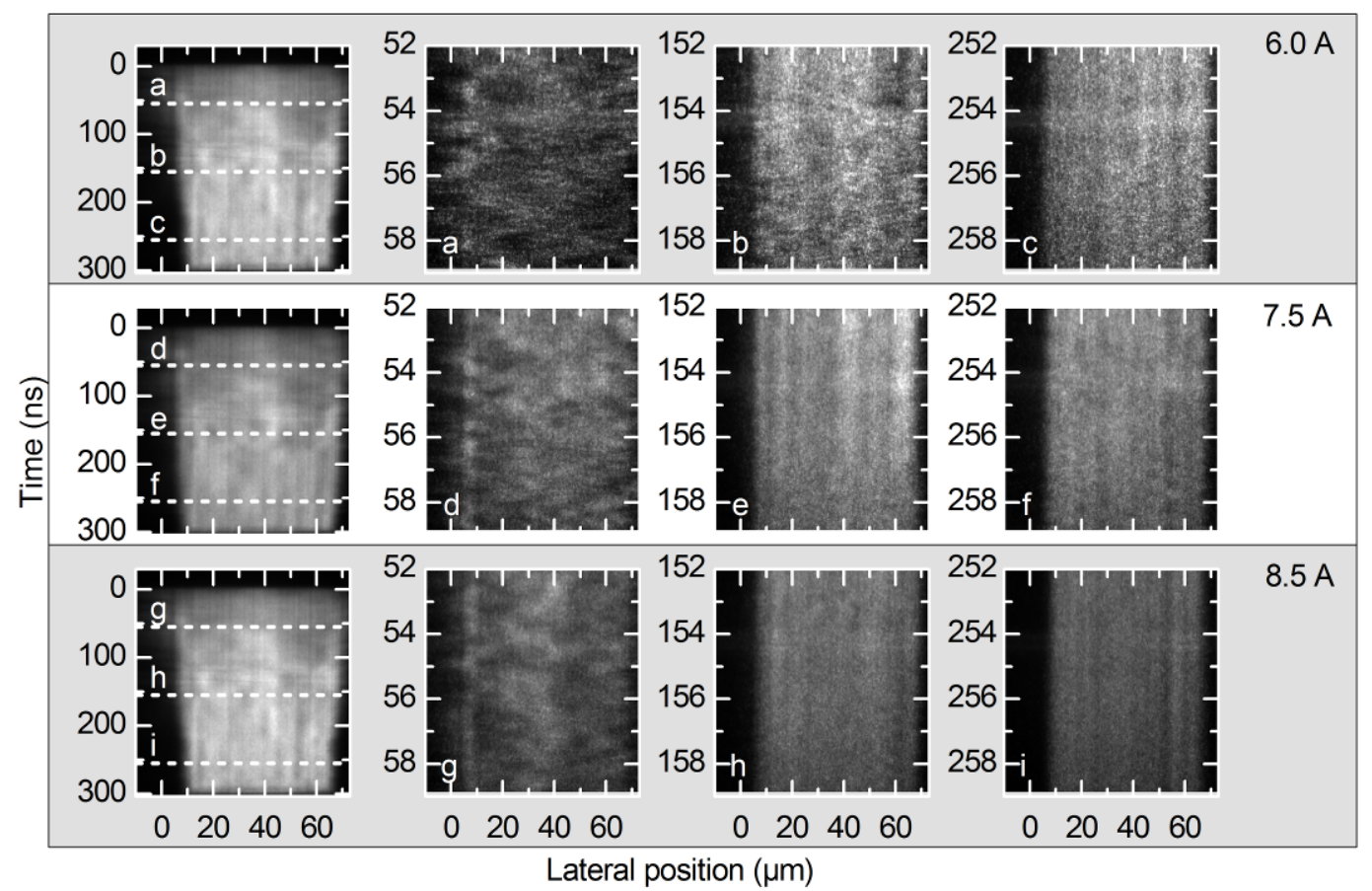

Figure 2. Typical NF evolution from the investigated batch of lasers. All images are taken from device A at driving currents as indicated. The left column shows overviews over the entire $300 \mathrm{~ns}$ pulses, while the other columns give the NF evolutions with improved temporal resolution at the indicated positions.

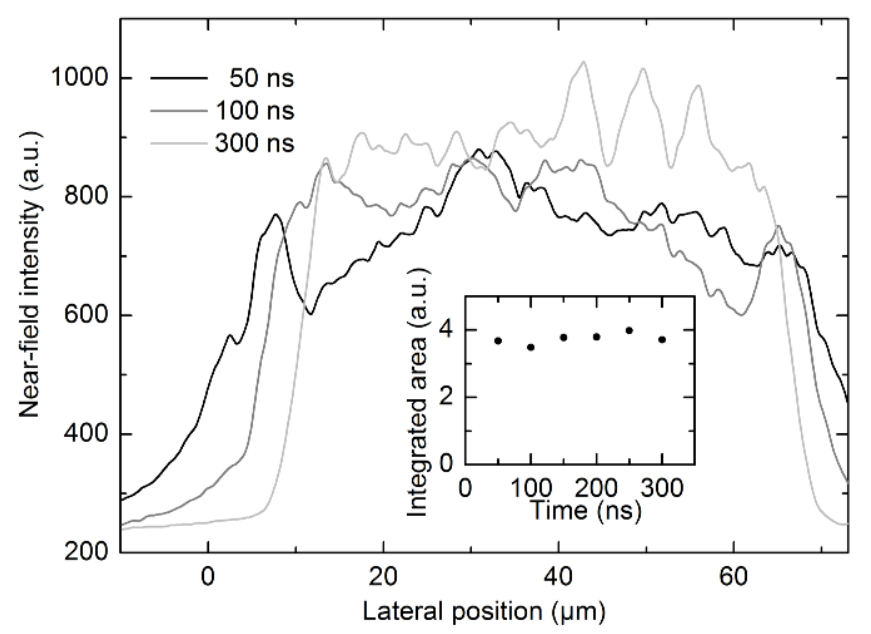

Figure 3. Time averaged NF's of device A, averaged over $\sim 8 \mathrm{~ns}$ around the indicated time in a $300 \mathrm{~ns}$ pulse at $8.5 \mathrm{~A}$. The inset gives the areas under the curves at different times. 


\subsection{Near-field en route to COD}

We follow the step test procedure, as described in section 2, until the COD threshold current is reached. The NF during device B's first COD event at 13 A driving current is shown in Fig. 4(a). The starting of COD related degradation is indicated by an arrow. The lateral spread of the region of reduced NF, the so-called $N F$ gap, with ongoing time is clearly visible. Figure 4(b) shows a comparison between the averaged NF before starting of COD, i.e. $<t_{\mathrm{COD}} \sim 230 \mathrm{~ns}$, and after. Subfigures (c) and (d) show longitudinal (along the laser axis) averaged lasing $(I=0.5 \mathrm{~A})$ and electroluminescence (EL, 0.1 A) NF's, respectively. They are taken with an optical microscope looking directly on the front facet, equipped with a camera that is set to a long integration time of $1.3 \mathrm{~s}$, resulting in a time averaged image. The agreement of the NF gap seen with the streak camera at the pulse end and the microscope analysis is evident.
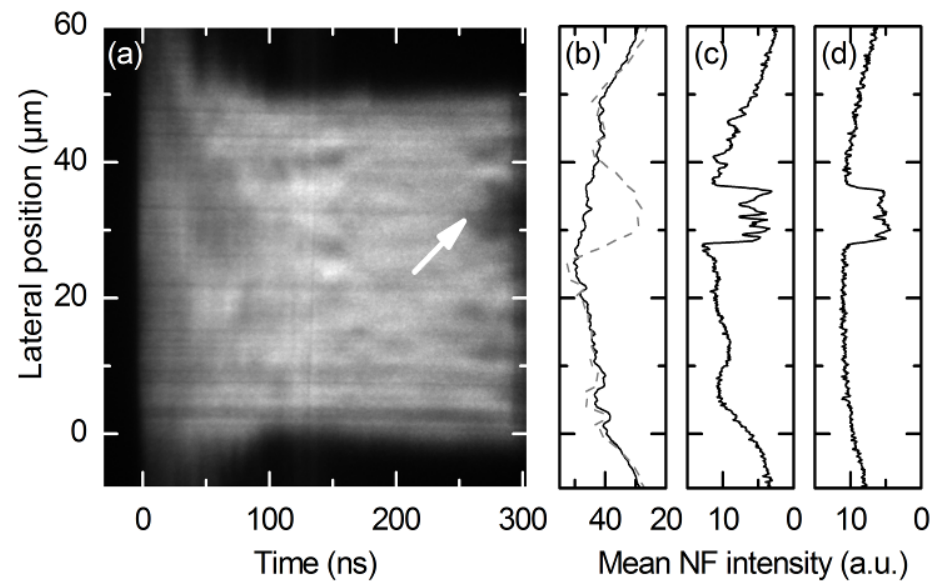

Figure 4. NF evolution of a COD event. (a) Streak camera image of NF from device B within the pulse where COD occurs at $I=13 \mathrm{~A}$. The spatial and temporal position of the first degradation sign is indicated by the arrow. The NF intensity is represented by the gray scale (dark = low intensity; light = high intensity) (b) Mean NF intensity before (black) and after (gray, dashed) the COD event (which takes place at $\sim 230 \mathrm{~ns}$ ). (c) Mean NF intensity taken from optical microcopy while applying $I=0.5$ A (lasing). (d) Mean NF intensity taken from optical microscopy under electroluminescence conditions at $I=0.1 \mathrm{~A}$ (spontaneous emission).

Figure 5(a) gives the optical output power development during the pulse where COD occurs for device $\mathrm{C}$ at $I=10 \mathrm{~A}$. This curve is directly compared with a curve extrapolated to $10 \mathrm{~A}$ based on the power transient recorded at lower currents during the step test. The reference curve represents the hypothetic situation without COD. The difference between them is indicated by a shaded area. Moreover the time $t_{\mathrm{COD}}$ from the leading edge of the pulse to the point where COD starts is marked.
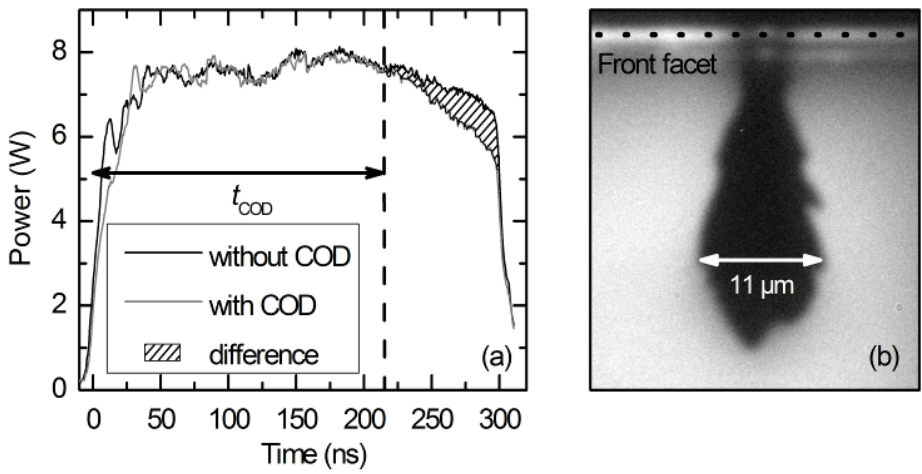

Figure 5. (a) Comparison of output power evolution for device $\mathrm{C}$ with or without a COD (curve without COD is scaled from power trace of previous current steps and confirmed by power traces of diode laser from the same batch with slightly higher COD threshold). COD takes place at $I=10 \mathrm{~A}$. The time to COD and the output power difference are indicated. (b) $\mu \mathrm{PL}$ image of the QW emission after COD and one COD re-ignition (together $\sim 270 \mathrm{~ns}$ of damage growth). The position of the front facet is indicated by the dotted line. 
After the end of the step tests a fraction of the batch is opened and a $\mu \mathrm{PL}$ image of the QW plane is taken. An example is shown in Fig. 5(b). The QW plane of device $\mathrm{C}$ is inspected from above. The position of the front facet is indicated. The marked maximal width of the damage site is confirmed by the NF monitored with streak camera and microscope; see Fig. 4(b-d).

\section{DISCUSSION}

\subsection{Thermal behavior}

We begin our discussion with the transient behavior of the NF at high pump currents. During the pulse, the width of the NF becomes significantly reduced; see Figs. 2 and 3. This is caused by the temperature rise of the active region. This temperature rise is quantified by two independent sets of experiments:

- First, the spectral shift of the laser emission is considered. Results for device D are shown in Fig. 6(a). The curve for $I=10$ A (see dashed line) is obtained by extrapolating the fitting curves for the measured values; see points taken at 1.6, 6, and $7 \mathrm{~A}$. Since $10 \mathrm{~A}$ corresponds roughly to the COD threshold of this device batch (when operated with $1 \mu$ s single pulses), this curve well describe the bulk temperature evolution during a pulse that leads to COD.

- Alternative information about the active region temperature is provided by the power drop. Figure 6(b) shows the emission power versus active region temperature as extrapolated from systematic measurements of the L-Icurves in the $20-80^{\circ} \mathrm{C}$ range. For 10 A pulses of $1 \mu$ s duration, we concordantly find an active region temperature increase of $(27 \pm 6) \mathrm{K}$.

The curve shown in Fig. 6(b) also explains why the steepest rise in bulk temperature does not lead to a dramatic change in the output power during the first $\sim 250 \mathrm{~ns}$, as one might expect. The power keeps nearly constant up to a temperature rise of $\sim 20 \mathrm{~K}$.

However, the measured active region temperature rise [Fig. 6(a)] leads, in accordance to the known ${ }^{11} \mathrm{~d} n / \mathrm{d} T$ relation, to a refractive index increase of $\Delta n=6.5 \times 10^{-3}$ after $300 \mathrm{~ns}$ at $I=10 \mathrm{~A}$. This causes the transition from pure gain-guiding to a thermally-induced index-guiding, as theoretically shown in Ref. ${ }^{12}$ In conclusion the output power remains nearly constant (see inset Fig. 3), while the width of the NF decreases and eventually the optical load at the facet increases.
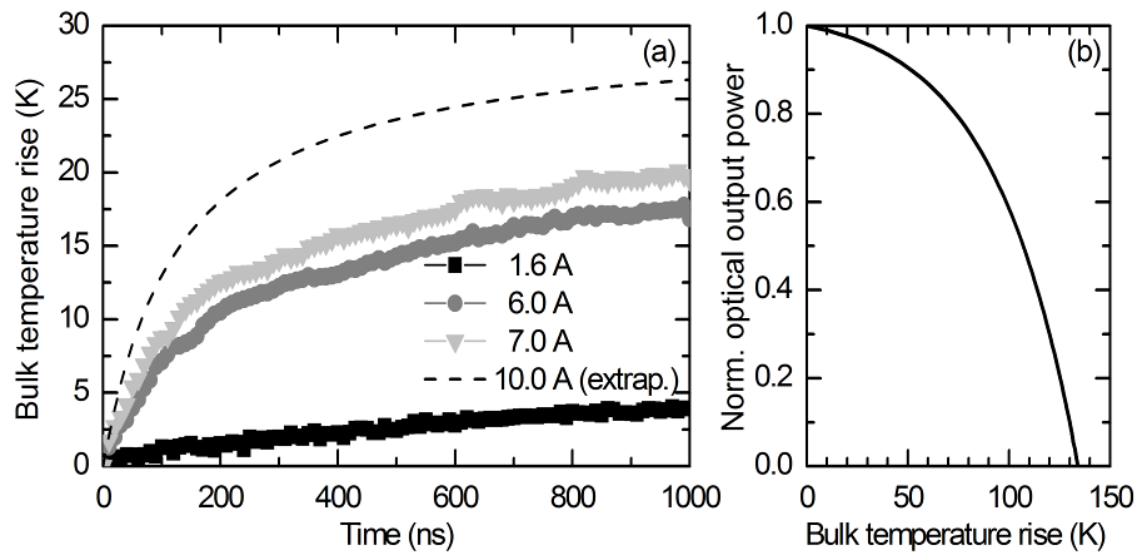

Figure 6. (a) Typical bulk-temperature evolution measured form device D. The temperature rise with respect to the heat sink temperature is shown. The temperature curve for $10 \mathrm{~A}$, a typical COD threshold current for the batch, is extrapolated from the fit curves of the measured data. (b) Normalized optical output power (normalized to $23^{\circ} \mathrm{C}$ value) at different bulk-temperature rises, extrapolated from temperature depended L-I-curves.

Figure 2 shows not only a reduction of the NF width, but also a fundamental change in its spatio-temporal structure. At the beginning, for lower current and shorter times, a clear zigzag motion is visible. The chaotic nature of this pattern is verified by averaging 100 consecutive taken NF images at the same temporal position and getting a 2-3 lobe NF, well known for the $\mathrm{cw}$ case of BA lasers. With increasing active region temperature the motion of the main filaments becomes imperceptible due to the temporal resolution of the setup., ${ }^{5,13}$ This is attributed to an increasing frequency of the spatio-temporal field dynamics, which is driven by the bulk temperature rise as well. ${ }^{14}$ 


\subsection{Damage propagation during the COD}

In the following the discussion is focused on two devices named B and C. While device B represents the devices with a slightly higher COD threshold, $\mathrm{C}$ represents an example for a device out of the center of the distribution. The statistical distribution of the COD threshold values is caused by small, not controllable inhomogenities in the material, which are always present.

From the streak camera images shown in Fig. 4(a), two important transient properties of devices B and C can be extracted and compared:

- the width of the NF gap, which gives the lateral damage spread, shown in Fig. 7(a) for devices B and C, including the data for the COD re-ignition provoked in case of device $\mathrm{C}$, and

- the reduction of NF intensity at the starting site of COD defect growth, Fig. 7(b).

Figure 7(a) shows that the starting point of damage creation in both devices is between $220 \mathrm{~ns}$ and $240 \mathrm{~ns}$. At the beginning of the process, a damage width of $\leq 1 \mu \mathrm{m}$ is created in less than $400 \mathrm{ps}$ and $\leq 2 \mu \mathrm{m}$ in less than $700 \mathrm{ps}$ in case of device B and C, respectively. This most likely represents the kinetics of thermal runaway as the fastest phase of the COD process, as described, e.g., in Ref. ${ }^{6}$ Thereafter the lateral damage expansion continues with a constant velocity of $(190 \pm 10) \mathrm{m} / \mathrm{s}$ for device B and $(30 \pm 3) \mathrm{m} / \mathrm{s}$ for device C. Device C has been now subjected to another current pulse, and a velocity of $(28 \pm 1) \mathrm{m} / \mathrm{s}$ is observed. This unchanged value shows that there is no significant reduction of the energy supporting the COD process. The obvious difference between the values for the two devices results from the different current at which COD starts. Whereas the value found for device $\mathrm{C}$ is quite common for diodes failing around $I=10 \mathrm{~A}$ throughout the whole batch, the velocity increases fast with increasing COD threshold current. To complete the line of arguments it is essential to consider the temporal evolution of the darkening of the NF at the COD starting site, shown in Fig. 7(b). It shows that the darkening in case of device $\mathrm{C}$ is faster up to $\sim 45 \mathrm{~ns}$ after COD starts. This remaining light intensity can be seen as a measure of the longitudinal dimension of the damaged region. A slower intensity decrease is interpreted as slower longitudinal defect growth. Concerning this it is clear that the internal damage geometry depends on the COD threshold current. While device $\mathrm{B}$ has a larger lateral damage growth rate, the longitudinal damage of device $\mathrm{C}$ develops faster as can be seen in the $\mu$ PL image Fig. 5(b).
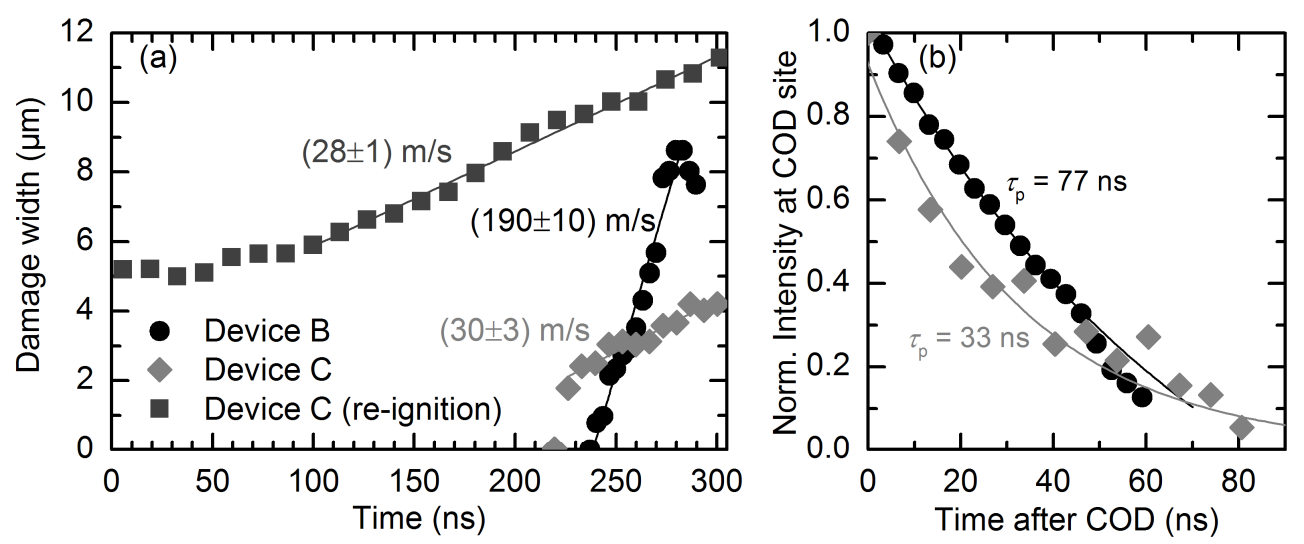

Figure 7. (a) Temporal evolution of the width of the NF gap during COD and in case of device C also during the re-ignition of the process. The linear fits and the resulting velocities of lateral motion are indicated. (b) Comparison of the normalized intensities (with respect to the average emission before COD) at the COD site (in the middle of the NF gap) of device $\mathrm{B}$ and $\mathrm{C}$. The zero point of the time axis is set to the respective $t_{\mathrm{COD}}$ values. The exponential decay curve fittings are indicated as lines and the characteristic decay times, $\tau_{\mathrm{P}}$ are given.

\subsection{The energy balance}

Our data allows an energy balance to be set up for the defect kinetics, as demonstrated below for device C.

A feasible assumption while investigating the system in a sub-microsecond regime is that the heat transport in the device can be neglected. As discussed in Sec. 4.2 the energy feeding the COD process keeps almost constant, which is valid as long as the damage width is small against the emitter width. Following this argument the difference in optical output 
power of a device with $\left(P_{\text {real }}\right)$ and without $\left(P_{\text {extra }}\right)$ COD gives the energy $W_{\text {diff }}$ consumed by the defect growth. This is illustrated in Fig. 5(a) and can be expressed as:

$$
W_{\text {diff }}=\int_{t_{C O D}}^{t_{\text {end }}}\left[P_{\text {extra }}(t)-P_{\text {real }}(t)\right] \mathrm{d} t
$$

integrating over the time from $t_{\mathrm{COD}}$ to the pulse end $\left(t_{\mathrm{end}}\right)$. This can also be done for the case of COD re-ignition in case of device C. Resulting in $W_{\text {diff,COD }}=45 \mathrm{~nJ}$ and $W_{\text {diff,follow }}=246 \mathrm{~nJ}$ for the COD pulse and the subsequent one, respectively.

These values can now be compared to the energy $W_{\text {melt }}$ needed to bring the affected material [as can be seen as dark region in Fig. 5(b)] to its melting temperature:

$$
W_{\text {melt }}=c \cdot \rho \cdot V \cdot \Delta T,
$$

with the temperature difference $\Delta T=1217 \mathrm{~K}$, taken from Ref. ${ }^{9}$, the mass density $\rho=5.33 \mathrm{~g} / \mathrm{cm}^{3}$, and the specific heat $c=$ $0.33 \mathrm{~J} /(\mathrm{g} \mathrm{K})$. The defect volume $V$ is the defect area $A_{\text {defect }}$ in the emitter plane times the waveguide thickness of $\sim 1 \mu \mathrm{m}$. The damaged area in the QW plane of device $\mathrm{C}$ after COD and COD re-ignition is known from the $\mu$ PL image, Fig. 5(b), as $A_{\text {dam, final }}=139 \mu \mathrm{m}^{2}$. It is necessary to divide this final area into a part $A_{\text {defect,COD }}$ created during the first COD event and $A_{\text {defect,follow }}$ created during COD re-ignition. Following the fact that the NF gap seen with the streak camera agrees well with the lateral defect dimension found in $\mu \mathrm{PL}$ and its linear growth, Fig. 7(a), $A_{\text {defect,final }}$ can easily be scaled to get $A_{\text {defect, } \mathrm{COD}}=23 \mu \mathrm{m}^{2}$ and $A_{\text {defect,follow }}=A_{\text {defect,final }}-A_{\text {defect, } \mathrm{COD}}=116 \mu \mathrm{m}^{2}$. Our calculations provide energies of $W_{\text {melt,COD }}=49 \mathrm{~nJ}$ and $W_{\text {melt,follow }}=248 \mathrm{~nJ}$, which are in very good agreement with the corresponding $W_{\text {diff }}$ values $\left(W_{\text {diff,COD }}=45 \mathrm{~nJ}\right.$ and $W_{\text {diff,follow }}=246 \mathrm{~nJ}$ ). Thus the loss in optical power is completely accumulated in the defect growth process. Again, this picture is only valid as long as the lateral damage width is small compared to the entire emitter width.

Furthermore, the energy needed to re-ignite the damage growth process can also be calculated. Figure 7(a) shows that the damage width for device $\mathrm{C}$ keeps constant during the first $100 \mathrm{~ns}$ before the lateral expansion re-starts. As demonstrated above the missing optical output power of $\sim 1 \mathrm{~W}$ is accumulated in the defect region, resulting in an energy of $\sim 100 \mathrm{~nJ}$. This is almost twice as high as the value needed to create the damage during the first COD event. The explanations for this difference are:

- In addition to the primary defect seen as dark region in the $\mu$ PL image, Fig. 5(b), a cloud of point defects around it is created by the COD process, as described in Ref. ${ }^{8}$ They absorb energy without heating the region around the existing primary damage to $T_{\text {crit, }}$, this energy gets lost in the balance calculations.

- After an initial defect is created in the laser cavity a redistribution of the lateral modes can be seen. This leads to an increased NF at so far undamaged parts of the facet ${ }^{8,9}$ resulting in a lower than expected optical power at the site of the main defect.

The second point implies also that the increased light field can create an additional COD starting point apart from the initial one. This defect spread mechanism depends on the light field and is therefore extremely fast compared to all other damage growth scenarios.

Moreover, it is possible to calculate the minimal current necessary to re-ignite the process during a $300 \mathrm{~ns}$ long pulse, which is $2.8 \mathrm{~A}$. Thus the $1.6 \mathrm{~A}$ test pulse applied is indeed not able to re-start the process. Whereas, in the case of a doubled defect width of $\sim 10 \mu \mathrm{m}$ (this is still significantly smaller than the emitter width) the absorbed energy would be sufficient for re-ignition. This means a critical combination of damage size and optical power level has to be considered to enable further degradation by COD. Especially, the presence of a defect site as absorbing element leads to a significant reduction of $t_{\mathrm{COD}}$ for further operation at a comparable power level. The calculations show that $t_{\mathrm{COD}}$ is mainly the time a certain volume around the COD starting point needs to reach $T_{\text {crit }}$.

\section{CONCLUSION}

In conclusion the experiments at extremely high pump levels show a clear change from pure gain to thermally-induced index guiding of the near-field intensity. Here the current and time dependent temperature of the active region is crucial. This change of the lateral mode regime leads to a higher optical load on the facet raising the local facet temperature. Additionally a considerable increase of the active material's bulk temperature can be measured. Fed by these two heat 
sources, a microscopic part of the facet (smaller than $2 \mu \mathrm{m}$ ) initially exceeds $T_{\text {crit }}$ and starts the COD defect growth process leading to further macroscopic damage sites.

The ratio between lateral and longitudinal defect growth is shown to depend on the COD threshold current. Lateral damage growth velocities ranging from approx. $30 \mathrm{~m} / \mathrm{s}$ to nearly $200 \mathrm{~m} / \mathrm{s}$ are shown. An upper time limit of $700 \mathrm{ps}$ can be given for the thermal runaway, as the fastest phase of COD, during which the initial defect is created.

\section{REFERENCES}

[1] R. L. Byer, "Diode-Laser - Pumped Solid-State Lasers," Science, 239(4841), $742-747$ (1988).

[2] S. R. Ahmad, and D. A. Russell, "Studies into Laser Ignition of Confined Pyrotechnics," Propellants Explosives Pyrotechnics, 33(5), 396-402 (2008).

[3] S. A. Payne, C. Bibeau, R. J. Beach et al., "Diode-pumped solid-state lasers for inertial fusion energy," Journal of Fusion Energy, 17(3), 213-217 (1998).

[4] M. O. Ziegler, M. Münkel, T. Burkhard et al., "Spatiotemporal emission dynamics of ridge waveguide laser diodes: picosecond pulsing and switching,” J. Opt. Soc. Am. B, 16(11), 2015-2022 (1999).

[5] I. Fischer, O. Hess, W. Elsasser et al., "Complex spatio-temporal dynamics in the near-field of a broad-area semiconductor laser," Europhysics Letters, 35(8), 579-584 (1996).

[6] J. W. Tomm, M. Ziegler, M. Hempel et al., "Mechanisms and fast kinetics of the catastrophic optical damage (COD) in GaAs-based diode lasers," Laser \& Photonics Reviews, 5(3), 422-441 (2011).

[7] M. Hempel, M. Ziegler, J. W. Tomm et al., "Time-resolved analysis of catastrophic optical damage in $975 \mathrm{~nm}$ emitting diode lasers," Applied Physics Letters, 96(25), 251105 (2010).

[8] M. Hempel, F. L. Mattina, J. W. Tomm et al., "Defect evolution during catastrophic optical damage of diode lasers," Semiconductor Science and Technology, 26(7), 075020 (2011).

[9] M. Ziegler, M. Hempel, H. E. Larsen et al., "Physical limits of semiconductor laser operation: A time-resolved analysis of catastrophic optical damage," Applied Physics Letters, 97(2), 021110 (2010).

[10] M. Baeumler, J. L. Weyher, S. Muller et al., [Investigation of degraded laser diodes by chemical preparation and luminescence microscopy] Institute of Physics Conference Series, Templin, Germany(1998).

[11]D. E. Aspnes, S. M. Kelso, R. A. Logan et al., "Optical Properties of $\mathrm{Al}_{\mathrm{x}} \mathrm{Ga}_{1-\mathrm{x}} \mathrm{As}$," Journal of Applied Physics, 60(2), 754-767 (1986).

[12] J. Mukherjee, and J. G. McInerney, "Spatial mode dynamics in wide-aperture quantum-dot lasers," Physical Review A, 79(5), 053813 (2009).

[13] H. Adachihara, O. Hess, E. Abraham et al., "Spatiotemporal chaos in broad-area semiconductor laser," J. Opt. Soc. Am. B, 10(4), 658-665 (1993).

[14] M. Hempel, J. W. Tomm, M. Baeumler et al., "Near-field dynamics of broad area diode laser at very high pump levels," AIP Advances, 1(4), 042148 (2011). 